http://jmscr.igmpublication.org/home/ ISSN (e)-2347-176x ISSN (p) 2455-0450 crossref DOI: https://dx.doi.org/10.18535/jmscr/v9i8.14

\title{
Management of 5 yrs old Midmandibular Soft Fluctuant Swelling Arising due to Buccal Cortical Perforation of Mandible Subsequent to Chronic Periapical Periodontitis of Carious Lower Central Incisors by a Combination of Surgical and Conventional Endodontic Approach with 20 Months Follow-Up
}

\author{
Authors \\ Dr Prashant Acharya ${ }^{1}$, Dr Neesha Kumari ${ }^{2}$, Dr Sanjivani Pandit ${ }^{3}$ \\ ${ }^{1}$ Regional Hospital Bilaspur Himachal Pradesh \\ ${ }^{2,3}$ Prakshroop dental clinic, Bilaspur Himachal Pradesh
}

\begin{abstract}
Inflammatory root canal infections become symptomatic and spread further towards alveolar bone predominantly due to collective pathogenicity of the multispecies community dominated by anaerobic bacteria like fusobacterium, prevotella, porphyromonas etc causing pathologies like apical periodontitis, periapical granulomas, abcesses and periapical cysts.

Nonsurgical and surgical endodontic treatments have a high success rate in treatment and prevention of apical periodontitis. Differential diagnosis of the periapical radioopacity is most important as these bone alternation could be neoplastic, dysplastic or of metabolic origin. So a detailed CBCT analysis of an inflammatory or non inflammatory lesion is necessary for the proper designing of treatment plan.

The advantages and shortcoming of the management approach while treating this case are discussed ahead.

Keywords: Intraosseous benign fibrocollagenous inflammatory growth, Apicoectomy, Apical root repair, $M T A$.

\section{Introduction}

Endodontic infection causes inflammation of the apical periradicular tissues manifesting as apical periodontitis. This inflammation can present itself in various clinical ways, it is also found to develop as an acute abscess. ${ }^{1}$

Nonvital pulp triggers the process of root canal infection. This infection proceeds due to degeneration of the dental pulp due to caries or traumatic injury to the tooth or if the pulp has been removed during earlier endodontic treatment. The infected root canal may send the bacteria and their noxious products through apical and lateral foramina connections or perforated root canals

inducing an acute or chronic inflammatory response. $^{2}$

The chronic inflammation causes bone around root apex to resorb with usually asymptomatic response and this resorption is the prominent radiographic manifestation of apical periodontitis. Contrary to this an acute apical inflammation manifests through pain and swelling as their typical signs and symptoms. The symptomatic illness may initiate acutely without established longstanding inflammation or may be a resultant aggravation of an old chronic asymptomatic illness. Only $5 \%$ cases has been estimated to exacerbate from asymptomatic chronic to symptomatic acute per year. $^{3}$
\end{abstract}


In advanced stages this chronic form of apical periodontitis presents as an acute abscess. In acute root canal infections the bacteria present in the canal spreads to periapical tissues and further beyond to other deeper planes of head and neck tissues to form a cellulitis presenting as a diffused spread of infection/inflammation with pus formation. ${ }^{4}$

Patient having acute apical abscess feels mild to intense pain and swelling or sometimes trismus also. The patient may be febrile having headache, malaise, nausea and may develop lymph node swellings as systemic presentation of the acute illness. Periapical bone destruction and resultant radiolucency in an IOPA may not be visible due to the fast spread of acute infection. When periapical radiolucency is observed in an IOPA x-ray, the abscess is an acute aggravation of a chronic infection (Fig. 1).Tenderness to percussion is mostly seen in the tooth involved. The exudate or the pus travels the path of least resistance through the medullary bone before perforating the cortical bone discharging it into soft tissues surrounding it. Swelling is usually intraoral but may be extraoral also. ${ }^{5}$

Root end location of the infected tooth determines the infectious spread of endodontic infection into the head and neck fascial spaces along with the thickness of the overlying bone and the muscle attachments in correspondence to the tooth apex. ${ }^{6}$

\section{Case Report}

A female patient aged 22 years presented to the outpatient dental department of a regional hospital with a complaint of fluctuant swelling of mid chin region since 5 years without any sinus or drainage extraorally (fig 3). On intra oral examination it was found that lower central incisiors were carious and tender to percussion. Radiographic examination of the region through IOPA showed periapical radiolucencies associated with both the teeth (fig 1). Initially following a conventional endodontic approach root canal treatment of both incisors was initiated. Access cavities were made and necrotic pulp debridement was done. Initial instrumentation was done using $2 \%$ hand $\mathrm{k}$ files till no. \#25 followed by rotary instrumentation by $4 \%$ edge files and BMP was done till no. \#30. Patient was then asked to report on weekly basis and irrigation with normal saline followed by closed dressing was done till a month. When no reduction in the size of extra oral swelling was seen an intracanal medicament metapex (calcium hydroxide + iodoform) was placed inside the root canals and IOPA radiographs were repeated. The sigmoid flow of the radioopaque intracanal medicament suggestive of an intrabony sinus tract and mandibular buccal cortical perforation leading to extraoral collection of exudates below the dermal tissues and the resultant fluctuant swelling was clearly visible in the IOPA radiographs (fig 2).

when no relief in pain and the size of the lesion was observed following 2 months of the treatment, a surgical approach was planned and a trapezoidal mucoperiosteal flap was raised from mandibular canine to canice region. Solidified pieces of intracanal medicament (metapex) along with subdermally accumulated inflammatory exudates were drained out and cortical perforation was cleaned off granulomatous tissue using bone curettes (fig 4).

Muscle tissue (mentalis) was also thoroughly debrided and after finding the extent of the lesion that involved cemental, periodontal ligament (PDL), bone and muscle tissue, a decision was made to extract both the central incisors to check any residual infections or further reinfection. A biopsy was conducted and the excised granulomatous tissues along with the apically resorbed central incisors were sent for histopathological examination. Biopsy of the test sample found it to be an inflamed fibrocollagenous tissue with walls showing dense mixed inflammatory infilterate comprised of polymorphs, lymphocytes, plasma cells and few macrophages with no atypia/ malignancy. Later when tenderness was found in lower right lateral incisor in a follow-up examination the root canal treatment of the concerned tooth was done. The patient was found to be completely asymptomatic in a 20 months long follow-up with complete bone healing and well formed bony trabecullaeas (Fig 7). 


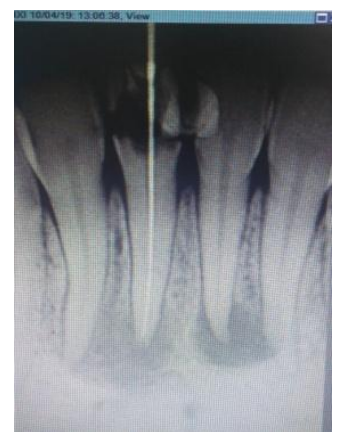

Fig 1. Initial IOPA

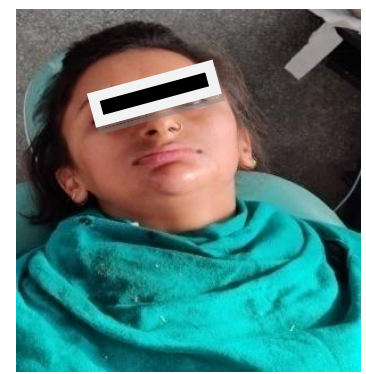

3. Pre-op patient photograph

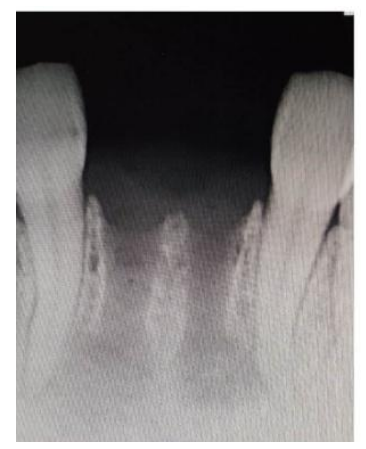

5. IOPA immediately after the procedure

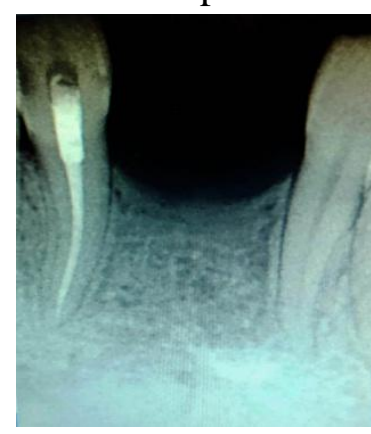

7. IOPA after

20 months

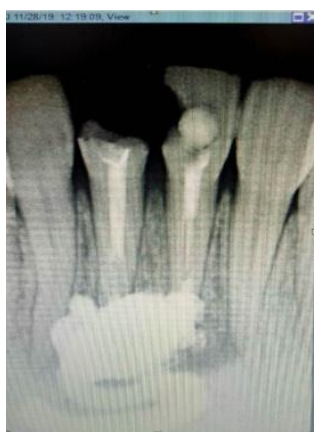

2. Metapex intracanal medicament in-situ

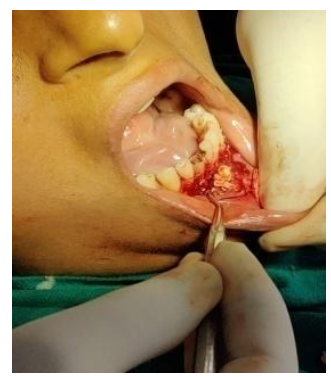

4. Surgical debridement of cortical perforation

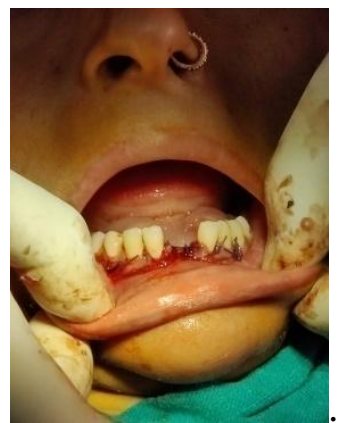

6. Immediate post-op surgical site

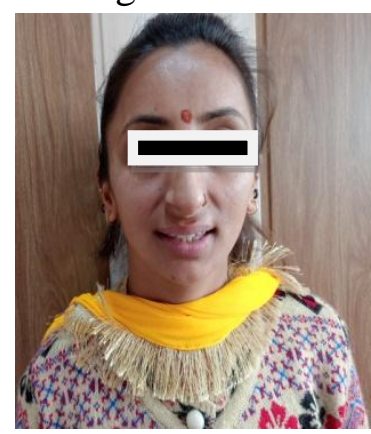

8. Patient photograph at 20 months

\section{Discussion}

Diagnosing a periradicular lesions is always challenging. ${ }^{7}$ As most of the pathologies seem similar to endodontic lesions even after being derivatives from different sources. ${ }^{8}$ Endodontic infection consequently causes Inflammatory modifications in the periradicular bone architecture due to changed osteoclastic or osteoblastic equilibrium. Radiolucent or radiopaque structures are the changes readily identified in x-ray images as a result of such modifications. ${ }^{9}$ Complement fixation and antigen antibody complex formation causes stimulation of cell rest of malassez and their resultant proliferation. Subsequent to it the growth factors like TGF-Beta, IL 1, IL6 and IL8 stimulates fibroblastic proliferation phase of the cell rest to form a periapical granuloma. ${ }^{10}$ Similarly, such bone modifications may also be there in pathologies of inflammatory, neoplastic, dysplastic and metabolic emergence. $^{11}$ Usually radiolucent lesions are commonly associated with infected root canals but radiopaque cases should also be viewed with doubt to check improper diagnosis. Whether these lesions are inflammatory or non-inflammatory pulp sensibility and vitality tests could be used to make a diagnosis. ${ }^{12}$ Usually a lack or delay of pulp response is seen in a periradicular lesion of endodontic origin, exceptionally this may too exist with a lesion of non-endodontic origin. Histopathological examination of the periradicular lesion or biopsy should be considered in cases where the clinical and radiographic findings seem to be insufficient in its identification. Ultrasonography, Orthopantamogram, Compuerised Tomography and Magnetic Resonance Imaging could also be used to study the extent of periradicular pathology. In this case the cost effective option of IOPA was chosen that could only reveal the medial and lateral extent. Anterio posterior dimensions of the lesion and its extent in axial, coronal and saggital planes remained undiagnosed and for that a computerised tomography was necessary. Other advantages of CT are

1) Fewer distortion of images

2) Physical tissue densities easily differentiated based on Hedstom units

3) No superimposition

4) No magnification

5) Better resolution. ${ }^{13}$ 


\section{Conclusions}

Thus our current case was managed by a combination of non surgical approach and surgical intervention. Asymptomatic operated oral tissues, the complete regression of the lesion extraorally and resumption of normal radioopacity of the operated region in a follow up of more than $1 \frac{1}{2}$ years are suggestive of buccal cortical bone repair. No use of CBCT in the present case is seen as one of the major drawback as the extent of cortical perforation and the intrabony portion of the lesion could not be three dimensionally analysed, due to limited resources and complaince of the patient.

\section{References}

1. Torabinejad M, Shabahang S. 2009. Pulp and periapical pathosis, p 49-67. In Torabinejad M, Walton RE (ed), Endodontics. Principles and practice, 4th ed. Saunders/Elsevier, St. Louis, MO.

2. Sasaki H, Stashenko P. 2012. Interrelationship of the pulp and apical periodontitis, p 277-299. In Hargreaves KM, Goodis HE, Tay FR (ed), Seltzer and Bender's dental pulp, 2nd ed. Quintessence Publishing, Chicago, IL.

3. Eriksen HM. 2008. Epidemiology of apical periodontitis, p 262-274. In Orstavik D, Pitt Ford $\mathrm{T}$ (ed), Essential endodontology, $2^{\text {nd }}$ ed. Blackwell Science Ltd, Oxford, United Kingdom

4. Baumgartner JC, Siqueira JF, Jr, Sedgley CM, Kishen A. 2008. Microbiology of endodontic disease, p 221-308. In Ingle JI, Bakland LK, Baumgartner JC (ed), Ingle's endodontics, 6th ed. BC Decker, Hamilton, Canada.

5. Gill Y, Scully C. 1990. Orofacial odontogenic infections: review of microbiology and current treatment. Oral Surg. Oral Med. Oral Pathol. 70:155-158.

6. Marcus BJ, Kaplan J, Collins KA. 2008. A case of Ludwig angina: a case report and review of the literature. Am. J. Forensic Med. Pathol. 29:255-259.
7. Estrela C, Holland R, Estrela CR, Alencar $\mathrm{AH}, \quad$ Sousa-Neto MD, Pécora JD. Characterization of successful root canal treatment. Braz Dent J. 2014;25(1):311.https://doi.org/10.1590/01036440201302356

8. Curé JK, Vattoth S, Shah R. Radiopaque jaw lesions: an approach to the differential diagnosis. Radiographics.2012;32(7):190925. https://doi.org/10.1148/rg.327125003.

9. Bender IB, Mori K. The radiopaque lesion: a diagnostic consideration. Endod Dent Traumatol. 1985;1(1):2-12. https://doi.org/10.1111/j.1600-

9657.1985.tb00551.x

10. Kusumi A, Sakaki H, Fukui R, Satoh H, Kusumi T, et al. (2004) High IL-6 synthesis in cultured fibroblasts isolated from radicular cysts. Arch Oral Biol 49: 643-652.

11. Fang Z, Jin S, Zhang C, Wang L, He Y. Misdiagnosis of osteosarcoma as cementoblastoma from an atypical mandibular swelling: a case report. Oncol Lett.2016;11(6):3761-5. https://doi.org/10.3892/ol.2016.4433

12. Bruno KF, Barletta FB, Felippe WT, Silva JA, Alencar AHG, Estrela C. Oxygen saturation in the dental pulp of permanent teeth: a critical review. J Endod. 2014;40(8):1054-7. https://doi.org/10.1016/j.joen.2014.04.01.

13. Frederiksen NL (2004) Specialized Radiographic Techniques. In: White SC, Pharoah MJ (eds) Oral Radiology: Principles and Interpratation. 245-250. 